\title{
Evaluation and Influencing Factors of Agricultural Eco-efficiency in Jilin Agricultural Production Zone Based on Super-SBM and Panel Regression Methods
}

\author{
Yanhua Guo \\ NENU: Northeast Normal University https://orcid.org/0000-0002-4313-4418 \\ Lianjun Tong ( $\nabla$ tonglj@iga.ac.cn ) \\ Northeast Institute of Geography and Agroecology Chinese Academy of Sciences \\ https://orcid.org/0000-0002-4339-9504 \\ Lin Mei \\ Northeast Normal University
}

\section{Research Article}

Keywords: Agricultural eco-efficiency, Spatiotemporal characteristics, Influencing factors, Super SBM-DEA, Agricultural production zone, Jilin Province

Posted Date: March 1st, 2021

DOI: https://doi.org/10.21203/rs.3.rs-225561/v1

License: (c) (i) This work is licensed under a Creative Commons Attribution 4.0 International License.

Read Full License 
1 Evaluation and influencing factors of agricultural eco-efficiency in Jilin agricultural production zone based on Super-SBM and panel regression methods

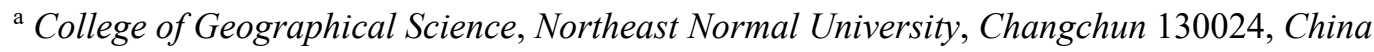

${ }^{\mathrm{b}}$ Northeast Institute of Geography and Agroecology, Chinese Academy of Sciences, Changchun 130102, China

Abatract Agricultural eco-efficiency is a meaningful index that assess the agricultural sustainable development. Based on the super SBM-DEA approach incorporating agricultural carbon emissions and panel data regression, this study evaluates agricultural eco-efficiency and then investigates the influencing factors in agricultural production zone of Jilin Province. The empirical results show the following: (1) During the observation period, the average agricultural eco-efficiency exhibits a flat "M-shaped" fluctuating trend, a trend of fluctuant growth with phase characteristics, and the agricultural eco-efficiency of each county still has much room for improvement. (2) Significant spatial differences exist in agricultural eco-efficiency across counties. All of the studied counties, except for Nong'an, Huadian, Lishu, Yitong, Gongzhuling, and Qianguo, need to change the input and output structure to optimize agricultural eco-efficiency. (3) The panel data regression model verifies that the agricultural technology extension level, agricultural economic development level, agricultural industry structure, agricultural mechanization intensity and urbanization level have close correlations with agricultural eco-efficiency. (4) The research findings have important implications for policy makers formulating agricultural environmental policies in accordance with 23 the local conditions of various counties. Keywords Agricultural eco-efficiency; Spatiotemporal characteristics; Influencing factors; Super SBM-DEA; Agricultural production zone; Jilin Province

\footnotetext{
${ }^{*}$ Corresponding author. Northeast Institute of Geography and Agroecology, Chinese Academy of Sciences, Changchun 130002, China.

${ }^{* *}$ Corresponding author. College of Geographical Science, Northeast Normal University, Changchun 130024, China.

E-mail address: tonglj@iga.ac.cn (L.-J. Tong), meil682@nenu.edu.cn (L. Mei).
} 


\section{Introduction}

The ecological and environmental issues caused by the continuous increase in the carbon emissions accompanying industrialization and urbanization have become increasingly prominent, attracting widespread attention from governments and scholars in various countries. Some countries, such as the UK and Japan, have put into practice actions and plans to achieve energy conservation and emission reduction. China, following the reform and opening up, has become the world's largest carbon emitter and has long regarded energy conservation and emission reduction as a national development strategy. In agriculture, China has made a significant achievement by feeding $20 \%$ of the world's population with $7 \%$ of the world's arable land, reaching a high enough level to satisfy the rising national demand for grain (Jin et al., 2019). Nevertheless, this achievement has actually resulted in prominent ecological environment issues, such as the degradation of cultivated land and organic material, the decrease in basic soil fertility, and the excessive consumption of agricultural chemical material. Agricultural carbon emissions accompany the unreasonable agricultural production activities, which makes agriculture the second main source of emissions in China. It must be recognized that the traditional agricultural production model characterized by high inputs, high consumption and low efficiency has become unsustainable. To respond to the severe carbon emissions associated with agriculture, the Chinese government has applied the strategy of zero growth in the usage of chemical fertilizers and pesticides, advocating the use of "grain storage in the land" and "grain storage in technology" to tackle practical problems. Moreover, agricultural subsidies have been provided to enhance agricultural production capacity and reduce agricultural production risk and encourage farmers to adopt practices to protect agricultural resources. To address these issues, this study applies agricultural carbon emissions as an indicator that affects the agricultural production environment for the study of agricultural eco-efficiency. The results may provide important practical guidance for achieving the dual goals of agricultural carbon emission reduction and the transformation of green agriculture.

The agricultural production zone in this study is perceived as a natural agricultural space occupied by cultivated land with advantageous agricultural conditions, high resource endowment and high productivity. In 2011, China officially issued the national policy of major function zoning, 
a national territorial development plan intended to achieve a sustainable geographic and landscape pattern (Fan and $\mathrm{Li}, 2009$ ). To manage the national territorial space, this plan creates a new organizational unit of regional functions (Wang and Fan, 2020), namely involving three spatial organizations: urbanization zone, agricultural development zone, as well as ecological security zone (Fan et al., 2012). For the agricultural development zone, the plan creates a new agricultural spatial organization with seven districts and twenty-three zones to ensure the safety of agricultural products in China. Specifically, as agricultural production zones are primarily oriented towards agriculture, their principal functions concern the effective supply of agricultural products and the comprehensive agricultural production capacity. With the gradual strengthening of resource and environmental constraints, the national agricultural economic development goals concern not only the improvement in grain production and the quantity of agricultural products but also the quality of agricultural economic growth to achieve a balance between the agricultural economy and resource supply. Accordingly, facing the severe constraints of the agricultural environment, how to promote agricultural eco-efficiency and achieve green agriculture is increasingly seen as the research frontier in geography and economics. In this context, how to coordinate the relationship between agricultural production and ecological civilization construction need to be studied in depth. A comprehensive study focusing on how influencing factors affect agricultural eco-efficiency over time would thus have great practical significance and could provide reference for the agricultural policy makers.

Jilin agricultural production zone, a crucial component of the Northeast agricultural production zone that is recognized worldwide for its advantages in commodity grain cultivation, has strategic significance for national grain security in China. Thus, it is adopted as our empirical study area. Facing the situation of resource restraints and increasing grain demand, what are the spatiotemporal characteristics of agricultural eco-efficiency in the Jilin agricultural production zone during the period 2005-2017? How do influencing factors accelerate or hider the enhancement of agricultural eco-efficiency in this agricultural production zone? What corresponding policy implications can be obtained from the empirical results to support policy makers? The findings regarding these key scientific issues can provide a theoretical reference with practical significance for accomplishing the country's carbon emission reduction targets ahead of schedule, optimizing the allocation of agricultural resources and promoting the green agricultural transition. 
The remainder of this study is organized in five sections. Section 2 reviews the relevant literature and the theoretical analysis on agricultural eco-efficiency. Section 3 introduces the study area, data sources, methodology and variables selection for empirical analysis. Section 4 provides an assessment of agricultural eco-efficiency, illustrates the spatial dynamics characteristic of agricultural eco-efficiency, and interprets the driving mechanism. Section 5 describes the relevant conclusions and proposes corresponding policy suggestions for policy makers.

\section{Literature review}

\subsection{Agricultural eco-efficiency}

Eco-efficiency emerged in the 1990s, when Schaltegger and Sturm (1990) was first proposed the concept as a quantitative tool of environmental management. In 1998, the Organization for Economic Co-operation and Development mentioned the concept of eco-efficiency with the propose of tackling the relationship between environmental impacts and agricultural production (Camarero, et al., 2013). Eco-efficiency conventionally refers to a process that seeks to maximize economic effectiveness while minimizing environmental impacts (Sinkin et al., 2008; Burnett et al., 2008).

Over time, eco-efficiency has been conceptualized and considered a topic of interest in an increasing range of fields (Reith and Guidry, 2003). The international and national research content of ecoefficiency is multi-dimensional and diversified (Zhang et al., 2008), covering the aspects of ecoefficiency evaluation (Huang et al., 2018; Czyżewski et al., 2019; Baum and Bieńkowski, 2020), the temporal evolution characteristics and spatial differentiation of eco-efficiency (Liu et al., 2020a; Chen et al., 2017), the influencing factors leading to changes in eco-efficiency (Moutinho et al., 2020), the evolution pattern of eco-efficiency, and strategies supporting the improvement in ecoefficiency. As the understanding of eco-efficiency grows, the number of related studies focusing on specific industries is gradually increasing. International and Chinese scholars have increasingly applied the lessons learned in the economic sector to other industrial sectors from different angles (Lio and Hu 2009). For the eco-efficiency evaluation of a specific industry, studies have gradually come to concentrate on specific kinds of eco-efficiency, considering eco-efficiency at the regional level (Zhou et al., 2020), in the tourism sector (Gössling et al., 2005; Liu et al., 2017; Peng et al., 2017), in various economic sectors (Xing et al., 2018), and in urban areas (Yin et al., 2014; Ren et 
al., 2019).

Among the in-depth studies on eco-efficiency, many have centered on agricultural production and grain security in the agricultural field. For instance, Picazo-Tadeo et al. (2011) assessed farming eco-efficiency applying data envelopment analysis (DEA) techniques. Gómez-Limón et al. (2012) evaluated the farm-level eco-efficiency among Andalusian olive farmers. Vlontzos et al. (2014) evaluated the agricultural energy and environmental efficiency of EU countries using the DEA approach. Todorovic et al. (2016) conducted the eco-efficiency assessment of agricultural water systems at the meso level by using the life-cycle system-based approach. Saravia-Matus et al. (2019) measured the relationship between greenhouse gas efficiency and agricultural production in the agricultural sector. Deng and Gibson (2019) estimated the agricultural eco-efficiency of Shandong in 1990-2010 based on stochastic frontier analysis. Liu et al. (2020b) estimated the agricultural ecoefficiency of Chinese provinces over the period 1978-2019. By applying the nonseparable hybrid DEA model considering undesirable outputs, Han and Zhang (2020) evaluated environmental efficiency and the total factor productivity of cultivated land use. Moreover, scholars of agricultural economics have made considerable efforts to explore the influencing factors that shape the spatiotemporal distribution characteristics of agricultural eco-efficiency. Gkiza and Nastis (2017) empirically verified the effect of human health on agricultural production efficiency. Czyżewski et al. (2020) examined the effect of the European Union Common Agricultural Policy on environmental sustainable value, confirming that the higher investment support and capital-labor ratio contributed to eco-efficiency. Coluccia et al. (2020) assessed the eco-efficiency of the Italian agricultural sector and demonstrated that the Common Agricultural Policy weakened the specific environmental externalities via environmentally friendly land use management. These studies apply a wide range of methods to comprehensively measure the eco-efficiency level, spatiotemporal characteristics and influencing factors of agriculture from different perspectives (Ma, et al. 2018a; Ma, et al. 2018b). Among the models used for assessment, the ecological footprint method (He et al., 2016; Yang and Yang, 2019), SFA and DEA are widely applied for the analysis of eco-efficiency, incorporating a multitude of input and output indicators. The major advantage of the DEA model over the SFA is that it can effectively eliminate the effect of random errors, and the function form need not be set in advance. Hence, various scholars have increasingly applied the DEA model to 
measure agricultural eco-efficiency. Additionally, various methods have been applied to verify the influencing factors of agricultural eco-efficiency. Among these methods, the panel data regression method and Tobit regression method are regarded as conventional instruments for analysis to identify the influencing factors. These models provide significant reference by revealing the temporal variation trends of agricultural eco-efficiency.

The previous research enriches the understanding of agricultural eco-efficiency, both theoretically and practically. However, empirical studies on agricultural eco-efficiency that focus on the main production area of agricultural products remain scarce, and spatially focused studies on agricultural production zones from the perspective of major function oriented zones are lacking. Accordingly, this study offers a potential contribution to the existing literature in two aspects. Existing studies have concentrated on the spatial dimension of agricultural eco-efficiency at the national level, provincial level, and city level, not the county level. Using the data available, this study aims to examine the spatiotemporal characteristics of agricultural eco-efficiency at the county level. In addition, in contrast with the traditional consideration of agricultural eco-efficiency that ignores resource and environmental factors, the improved assessment of agricultural eco-efficiency in this study accounts for the negative impact of resource constraints to accurately reflect the performance of agricultural economic growth. As such, this study not only reveals the characteristics of agricultural eco-efficiency over time and space but also estimates the potential influencing factors to propose suggestions for policy makers and agricultural managers.

\subsection{Analytical framework}

Under the interactions among the economic, social and environmental systems, different agricultural production conditions and human development factors are intertwined, complicating the change process of the spatiotemporal pattern of agriculture eco-efficiency. One the one hand, the ratio and scale of agricultural input and output directly affect agricultural eco-efficiency. Agricultural practitioners, the actual implementers of modern agricultural production, determine the amount and structure of input factors, such as land use structure, planting structure, farmland management scale, farming methods, and level of production. Therefore, with the conversion between input and output, changes in input-output structure can directly cause changes in 
agricultural eco-efficiency by affecting the allocation and utilization of resources for agricultural production. On the other hand, agricultural eco-efficiency is also indirectly affected by changes in external socioeconomic conditions. For example, economic development level, the transfer of rural laborers to cities, agricultural policies and agricultural market conditions also significantly affect agricultural eco-efficiency at the macro level. This study intends to explore the complex relationship between agricultural production and agricultural eco-efficiency by considering both its direct and indirect influences. To support this in-depth understanding, an analytical framework illustrating the interactions between agricultural production and agricultural eco-efficiency is proposed in Fig. 1.
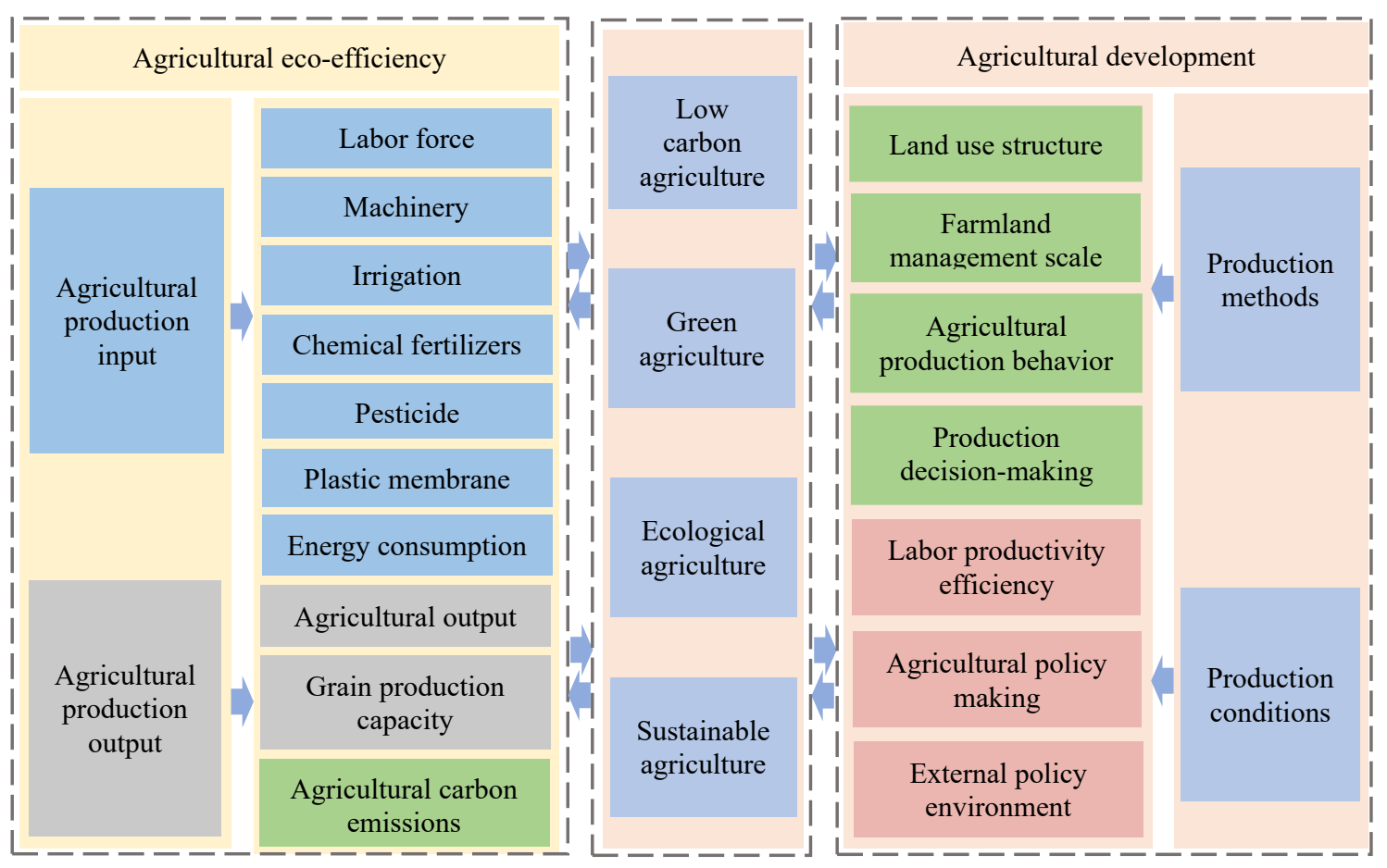

Fig. 1 An analytical framework illustrating the interactions between agricultural production and

\section{Data, methodology and variable selection}

\subsection{Study area and data sources}

This study adopts the agricultural production zone of Jilin Province (JAPZ) as the empirical study area. According to the Major Function Oriented Zone Plan of China and Jilin Province, 28 counties in Jilin form this major grain production zone, covering $102,598.59 \mathrm{~km}^{2}$ and occupying 
the total population in the province, the JAPZ generated 808.319 billion yuan $(57.48 \%)$ of the province's GDP in 2018. Notably, given the lack of statistical data, Shuangyang District and Jiutai District of Changchun City and Taobei District of Baicheng City are not considered in our empirical study. This study evaluates the agricultural eco-efficiency at the county level and estimates its influencing factors by employing a panel dataset composed of 26 counties during the 2005-2017 period. Fig. 2 presents a map of the empirical study area. Original socioeconomic data are compiled from the Statistical Yearbooks of Jilin Province, Changchun City, Jilin City, Siping City, Songyuan City, Baicheng City, Liaoyuan City, and Tonghua City for 2006-2018.

197
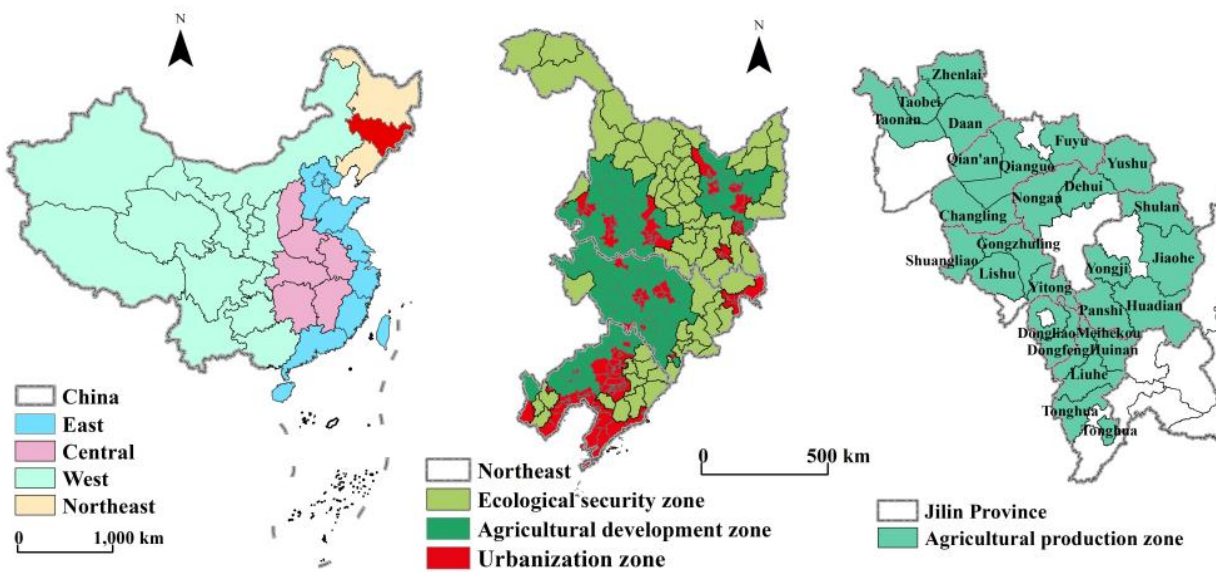

A

Fig. 2 Map of the empirical study area.

\subsection{Variables selection}

\subsubsection{Dependent variable: agricultural eco-efficiency}

The underlying principle of agricultural eco-efficiency is to create agricultural economic value with less agricultural input while continuously reducing the effects on the ecology and natural environment. Thus, the calculation of agricultural eco-efficiency integrates three dimensions: production inputs, desirable outputs and undesirable outputs. Table 1 displays the agricultural ecoefficiency evaluation index system. Specifically, a conventionally used strategy is established to measure the production factor inputs using the indicators of labor force, machinery, energy, irrigation, chemical fertilizers, pesticide, and plastic membrane. The desirable outputs comprise two types of agricultural output in the agricultural production zone, namely, agricultural output and grain production capacity. For the undesirable outputs, this study employs the amount of agricultural carbon emissions as the proxy measure. Referring to previous scholarly work (Tian et al., 2014; 
211 West and Marland 2002), this study selects the agricultural chemical fertilizers, pesticides, plastic

212 sheeting, diesel oil, irrigation, and tillage as the carbon sources of agricultural production activities.

213 Their emission coefficients are $0.8956(\mathrm{~kg} / \mathrm{kg}), 4.9341(\mathrm{~kg} / \mathrm{kg}), 5.18(\mathrm{~kg} / \mathrm{kg}), 0.5927(\mathrm{~kg} / \mathrm{kg})$,

$214266.48\left(\mathrm{~kg} / \mathrm{hm}^{2}\right)$ and $312.6\left(\mathrm{~kg} / \mathrm{km}^{2}\right)$, respectively. We multiply the emission coefficients by the

215 usage amount or acreage to calculate the total agricultural carbon emissions (Dubey and Lal, 2009;

216 Huang et al., 2019). Table 2 presents a statistical description of the indexes used for assessing

217 agricultural eco-efficiency.

218 Table 1 Agricultural eco-efficiency evaluation index system.

\begin{tabular}{|c|c|c|}
\hline Category & Specific Indication & Description \\
\hline \multirow{7}{*}{$\begin{array}{l}\text { Productio } \\
\mathrm{n} \text { inputs }\end{array}$} & Labor force & Total agricultural labor per unit of cultivated land \\
\hline & Agriculture machinery & Total agricultural machinery power per unit of cultivated land \\
\hline & Energy consumption & Amount of agricultural diesel consumption per unit of cultivated land \\
\hline & Irrigation & Effective irrigation area per unit of cultivated land \\
\hline & Chemical fertilizers & Amount of agricultural chemical fertilizers per unit of cultivated land \\
\hline & Pesticide & Amount of agricultural pesticide usage per unit of cultivated land \\
\hline & Plastic membrane & Amount of agricultural plastic membrane per unit of cultivated land \\
\hline \multirow{2}{*}{$\begin{array}{l}\text { Desirable } \\
\text { outputs }\end{array}$} & Agricultural output & Total gross output value of agriculture per unit of cultivated land \\
\hline & $\begin{array}{l}\text { Grain production } \\
\text { capacity }\end{array}$ & Total grain output \\
\hline $\begin{array}{l}\text { Undesirab } \\
\text { le output }\end{array}$ & $\begin{array}{l}\text { Agricultural carbon } \\
\text { emissions }\end{array}$ & Total amount of agricultural carbon emissions per unit of cultivated land \\
\hline
\end{tabular}

Table 2 Descriptive statistics of the agricultural eco-efficiency assessment indexes.

\begin{tabular}{llllll}
\hline Indexes & Unit & Min & Max & Mean & Std. dev \\
\hline Labor force & person/hm $/ \mathrm{hm}^{2}$ & 0.34 & 2.45 & 0.957 & 0.38 \\
Agriculture machinery & $\mathrm{KW} / \mathrm{hm}^{2}$ & 1.13 & 8.52 & 4.333 & 1.33 \\
Energy consumption & $\mathrm{kg} / \mathrm{hm}^{2}$ & 30 & 536 & 116.84 & 57.98 \\
Irrigation & $\%$ & 4.23 & 91.7 & 34.36 & 19.76 \\
Chemical fertilizers & $\mathrm{kg} / \mathrm{hm}^{2}$ & 174 & 2274 & 766 & 207 \\
Pesticide & $\mathrm{kg} / \mathrm{hm}^{2}$ & 1.32 & 107.53 & 10.28 & 9.56 \\
Plastic membrane & $\mathrm{kg} / \mathrm{hm}^{2}$ & 1.49 & 165.1 & 11.19 & 12.88
\end{tabular}




$\begin{array}{lllccc}\text { Agricultural output } & 10^{4} \mathrm{yuan} / \mathrm{hm}^{2} & 0.86 & 10.26 & 3.94 & 1.69 \\ \text { Grain production capacity } & \mathrm{kg} / \mathrm{hm}^{2} & 2.88 & 10.94 & 6.67 & 1.56 \\ \text { Agricultural carbon emissions } & \mathrm{kg} / \mathrm{hm}^{2} & 399 & 2352 & 958.99 & 235.69\end{array}$

\subsubsection{Independent variable selection}

(1) Agricultural machinery intensity $(A M I)$. Because high-intensity agricultural machinery can efficiency is unknown. Thus, this study employs the ratio of the total mechanical power to the crop sown area as the proxy variable of agricultural machinery intensity.

(2) Multiple-crop index (MI). The multiple-crop index refers to the frequency of planting crops per unit of cultivated land. This study uses the proportion of the crop sown area to the cultivated land area as the proxy variable of the multiple-crop index.

(3) Scale of family farmland management $(F F M S)$. Agricultural eco-efficiency also affects the environment through the expansion of the family farmland management scale. As the scale of production and operation units reaches an appropriate level, the allocation of production factors achieves the best operating efficiency, which leads to changes in production, life, ecology and indirectly guide agricultural production in a more environmentally friendly direction. Due to the unavailability of related data in the statistical yearbooks, the number of agricultural professional and technical personnel is applied as the proxy variable of the agricultural technology extension level.

(5) Agricultural economic development level (AEDL). The agricultural economic development level may have a close relationship with agricultural environmental quality according to the theory therefore applies the per capita agricultural output value to represent the agricultural economic development level.

(6) Agricultural industrial structure (AIS). Compared with the non-planting industries, planting 
industries may have a greater impact in the agricultural ecological environment through high labor input and labor intensity. Thus, this study applies the proportion of the planting industry in the primary industry as the proxy variable of the agricultural industrial structure.

(7) Urbanization level $(U L)$. The transfer of the non-agricultural population can lead to changes in agricultural eco-efficiency. Thus, the urbanization level, which here refers to the urban population as a percentage of the total population, is employed as the proxy variable of the urbanization level.

(8) Level of rural resident income (RRIL). High resident income generates an income effect and a substitution effect on agricultural eco-efficiency and then prompts rural residents to increase the input of production factors, inevitably resulting in agricultural emissions. However, an improvement in rural resident income level may enable famers to afford high-quality production factors, thereby decreasing agricultural pollution. The per capita net income of rural residents is regarded as the proxy variable of rural resident income level.

\subsection{Methodology specification}

\subsubsection{Measuring agricultural eco-efficiency: Super SBM-DEA}

The data envelopment analysis (DEA) model proposed in 1978 is an extensively used linear programming technique that can effectively evaluate the relative efficiency of decision-making units (DMUs). The conventionally used DEA model contains the CCR-DEA model and the BCC-DEA model. The former supposes that the returns to scale are constant (Charnes et al., 1978), while the later supposes that the returns to scale are variable (Banker et al., 1984). Both of these conventional DEA models are radial and oriented and thus overestimate efficiency. As such, Tone (2001) proposed a non-radial and non-oriented slack-based model (SBM) able to account for slackness, which can directly overcome the input and output slacks in the measurement. However, it is possible for multiple DMUs to have valid effective status denoted by $100 \%$ at the same time, which makes it difficult to rank and compare the efficiency of DMUs (Färe et al., 1989). To address this issue, Tone (2002) extended the model, proposing the super SBM-DEA model to innovatively solve these disadvantages of the traditional SBM-DEA model. The super-SBM method can provide a clear ranking based on the effective agricultural eco-efficiency scores (Li et al., 2013). Thus, this study applies the counties as the DMUs of the agricultural development frontier, using the data for 26 
counties in 2003-2016. The formula of the super SBM-DEA model is shown as follows:

$$
\begin{gathered}
\min \rho=\frac{\frac{1}{m} \sum_{i=1}^{m} \frac{s_{i}^{-}}{x_{i k}}}{\frac{1}{s_{1}+s_{2}}\left(\sum_{r=1}^{s_{1}} \frac{s_{r}^{g}}{y_{r k}^{g}}+\sum_{q=1}^{s_{2}} \frac{s_{r}^{b}}{y_{q k}^{b}}\right)} \\
\text { s.t. }\left\{\begin{array}{l}
s_{i}^{-} \geq \sum_{j=1, \neq k}^{m} \lambda_{j} x_{i j}, \quad s_{r}^{g} \leq \sum_{j=1, \neq k}^{n} \lambda_{j} y_{r j}^{g}, \quad s_{r}^{b} \geq \sum_{j=1, \neq k}^{n} \lambda_{j} y_{q j}^{b} \\
s_{i}^{-} \geq x_{k}, s_{r}^{g} \leq y_{k}^{g}, s_{r}^{b} \geq y_{k}^{b}, \lambda_{j} \geq 0
\end{array}\right.
\end{gathered}
$$

Where objective function $\rho$ is the agricultural eco-efficiency, and its variation range can be more than 1 ; if $\rho=1$, and the $s_{m}^{x}, s_{n}^{y}, s_{i}^{b}=0$, the DMU is effective, if $0 \leqslant \rho<1$, the DMU is ineffective, and the input and output should be improved. $\lambda_{j}$ denotes the coefficient, $m$ is the number of input indicators, $s_{1}$ is the number of desirable output indicators, $s_{2}$ is the undesirable output indicators, $s_{i}$, $g_{r}^{g}$, and $s_{r}^{b}$ are the slack variables, and $x_{i k}, y_{r k}^{g}$, and $y_{q k}^{b}$ denote the ith input, the $r t h$ desirable output and the $q$ th undesirable output value of county $k$. It is noted that the super SBM-DEA model assumes that there are constant returns to scale.

\subsubsection{Verifying the influencing factors: Panel data regression model}

Panel data, also called time series and cross-sectional data or pooled data, are two-dimensional data obtained over time and across space (Zhou et al. 2018). The panel data regression method can simultaneously reflect the changing pattern and characteristics of variables across the two dimensions of time and space, control individual heterogeneity and endogeneity problems, and improve the effectiveness of parameter estimation. Therefore, this method is widely used for modeling economic problems. The model is defined as the following formula:

$$
Y_{\mathrm{it}}=\beta_{0}+\beta_{1} X_{i t}+\beta_{2} X_{i t}+\beta_{3} X_{i t}+\ldots+\beta_{n} X_{i t}+\beta_{n+1} X_{i t}+\varepsilon_{i t}
$$

where $Y_{i t}$ is the dependent variable, $X_{i t}$ is the independent variable, $\beta_{0}$ denotes the constant, $\beta_{1}, \beta_{2}, \ldots$ $\beta_{n}, \beta_{n+1}$ represent the regression parameters, $\varepsilon$ is the random error, $i$ represents the county and $t$ denotes the time.

To eliminate the heteroscedasticity of variables, we take the natural logarithm of the original data for further conducting the panel data regression model. 
where the variables $A M I, X M I, F F M S, \ldots, U L, R R I L$ have the same implications as in Section 3.2.2 and in formula (3). Table 3 presents the descriptive statistics of the dependent and independent variables used in this empirical study.

Table 3 Descriptive statistics of dependent and independent variables.

\begin{tabular}{lllllll}
\hline Variables & Simple & Unit & Min & Max & Mean & Std. dev \\
\hline Agricultural eco-efficiency & AEE & - & 0.18 & 1.59 & 0.74 & 0.35 \\
Agricultural machinery intensity & AMI & KW/hm & 1.13 & 8.52 & 4.33 & 1.33 \\
Multiple-crop index & MI & - & 0.72 & 1.37 & 1.03 & 0.1 \\
Scale of family farmland management & FFMS & $\mathrm{hm}^{2} /$ house & 0.52 & 3.39 & 1.53 & 0.59 \\
& & hold & & & & \\
Agricultural technology extension level & ATEL & Person & 140 & 5473 & 1215 & 1127 \\
Agricultural economic development level & AEDL & Yuan & 2277 & 72192 & 9766 & 7716 \\
Agricultural industrial structure & AIS & $\%$ & 22.14 & 86.15 & 50.58 & 11.34 \\
Urbanization level & UL & $\%$ & 10.01 & 83.65 & 32.42 & 13.65 \\
Level of rural resident income & RRIL & yuan & 2244 & 13587 & 7427 & 3205 \\
\hline
\end{tabular}

304

\section{Empirical results}

According to the abovementioned model specification, this study evaluates the agricultural eco-efficiency using the super SBM-DEA approach that incorporates agricultural carbon emissions and then investigates its influencing factors using the panel data regression method.

\subsection{The temporal variation characteristics of agricultural eco-efficiency}

Based on formula (2) and the variables selected in Section 3.2.1, which include the indicators for seven inputs and three outputs, agricultural eco-efficiency scores are derived by operating MATLAB (R2016a) software. Fig. 3 illustrates the temporal variation characteristics of the average agricultural eco-efficiency of 26 counties in the agricultural production zone of Jilin from 2005 to 2017. During the observation period, the temporal variation characteristics of the average agricultural eco-efficiency values exhibit a flat "M-shaped" fluctuation trend. The agricultural ecoefficiency of the agricultural production zone shows a trend of continuous growth with fluctuation and is characterized by obvious periodic features. Specifically, the average agricultural eco- 
efficiency first increases steadily with a low growth rate from 2005-2010. This is mainly associated with the series of measures implemented to address the three rural issues associated with agriculture, rural areas, and rural peasants and the tax reduction and exemption policies enacted to support the continuous transformation of traditional agriculture to modern agriculture. However, agricultural eco-efficiency then begins to decline with rapid speed from 2011-2012, showing a trend of decrease by a wide margin. In this period, the government paid more attention the "urban disease" accompanying rapid urbanization development, and the counties' environmental governance in the agricultural sector became looser in the absence of specifically targeted guiding policies for the agricultural sector compared with the urbanization sector. In 2012, the score of agricultural ecoefficiency hit the lowest point of only 0.609. Then, agricultural eco-efficiency bounced back again in 2013-2014, probably because with the implementation of the ecological agricultural production model, the government paid more attention to agricultural resource constraints and strengthened the agricultural policy incentives. In 2015-2016, agricultural eco-efficiency slowly declined again, and it finally increased in 2017. This may be because of the low usage rate of agricultural chemical material, more attention to the agricultural ecosystem, and the high average agricultural ecoefficiency in these years. Overall, the average agricultural eco-efficiency of each year in the agricultural production zone of Jilin was approximately 0.689 , varying from 0.609 to 0.766 , which is an average level and indicates that there is much room for improvement in agricultural development even though the agricultural eco-efficiency indicates good capacity.

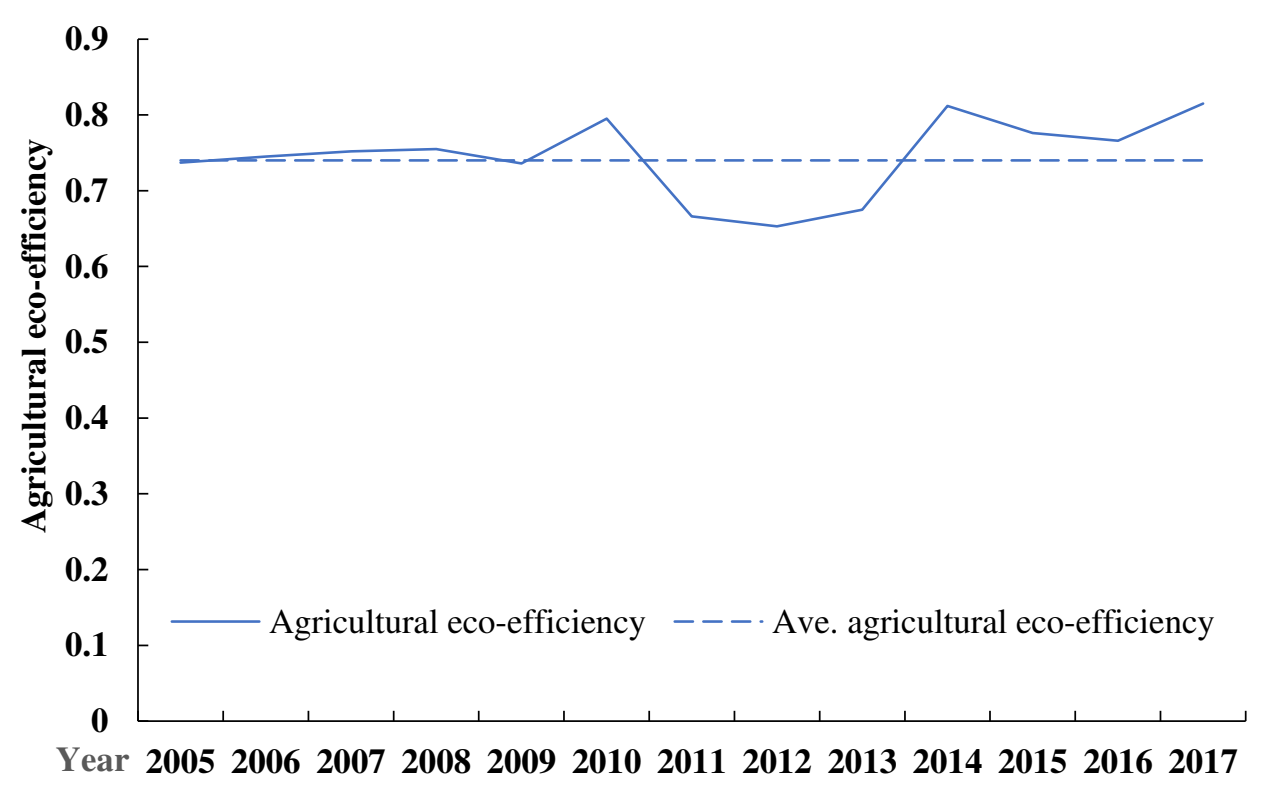




\subsection{The spatial distribution characteristics of agricultural eco-efficiency}

Fig. 4 plots boxplot of the agricultural eco-efficiency of 26 counties in 2006-2017. Noticeably, the average agricultural eco-efficiency of Lishu reached 1.32, the highest level, while the values for Yitong, Huadian, Gongzhuling, Nong' an and Qianguo averaged approximately 1.25, 1.18, 1.05, 1.09 , and 1.1, respectively. In addition, the variance of the agricultural eco-efficiency values in Changling, Qian'an, Tonghua, and Dehui is large, which demonstrates that the agricultural ecoefficiency of these counties has large gaps in the efficiency values, and the agricultural ecoefficiency is in an unstable state of fluctuation. In contrast, Meihekou, Taobei, Yongji and Taonan, all of which have weak resource carrying capacity, had very low agricultural eco-efficiency values. For the western counties with the lowest agricultural eco-efficiencies, namely, Taobei, and Taonan, this is primarily due to their geographical location with barren saline soil and water shortage, such that they require higher production inputs than other counties. For the central and eastern counties with the lowest agricultural eco-efficiencies, namely, Meihekou and Yongji, the low agricultural eco-efficiency values owe primarily to the inappropriate terrain and incomplete water conservancy facilities. The variance of agricultural eco-efficiency values in Yongji, Jiaohe, Dongfeng, Huinan, Liuhe, Meihekou, Zhenlai,Taonan, Daan, Taobei, is small but their agricultural eco-efficiency level is low, illustrating the relative bad stability of the agricultural eco-efficiency level in these counties and indicating the severity of the long-term inefficiency. In contrast, the variance of Nong'an, Huadian, Lishu, Yitong, Gongzhuling, Shuangliao and Qianguo is small and their agricultural ecoefficiency level is high, illustrating the relative good stability of the agricultural eco-efficiency level. 


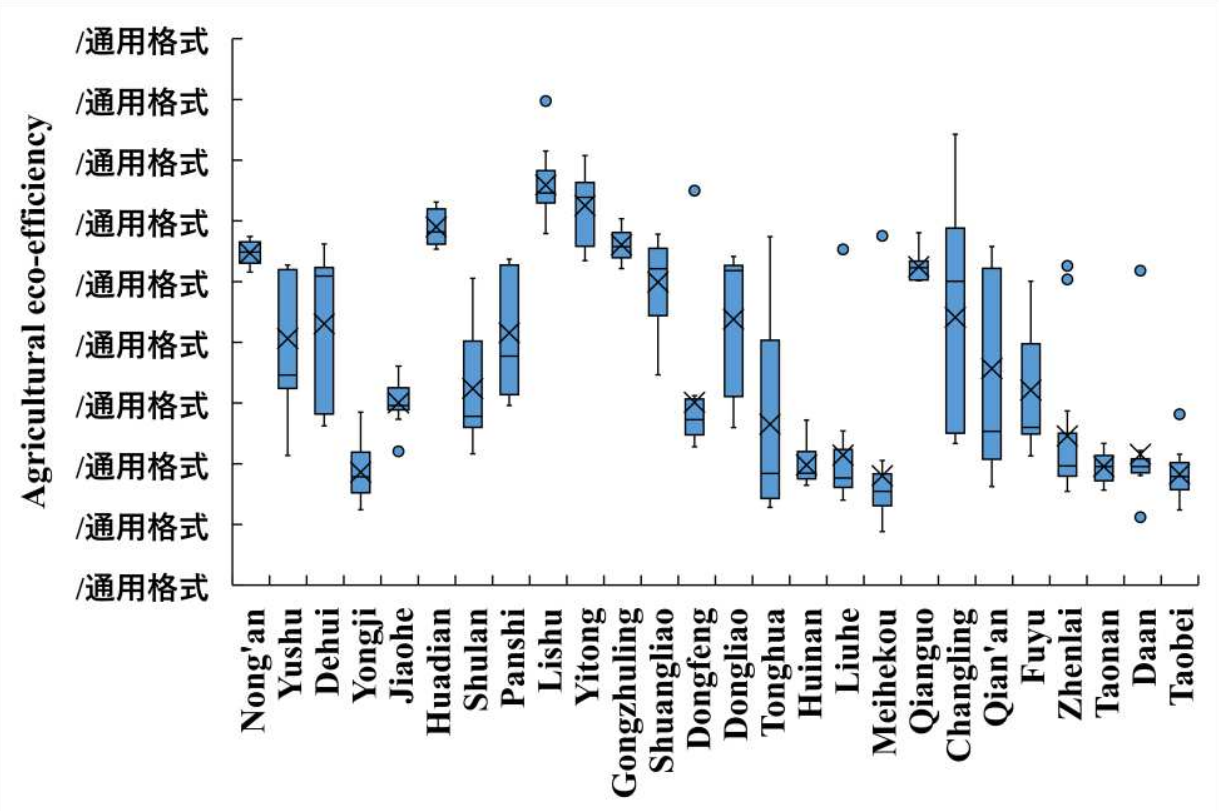

Fig. 4 Boxplot of the agricultural eco-efficiency of 26 counties in the JAPZ, 2005-2014.

To better observe the spatiotemporal characteristics of agricultural eco-efficiency, this study, based on ArcGIS 10.5 software, categories the agricultural eco-efficiency scores calculated by the super-SBM method into five levels: low level (0 0.30), medium-low level (0.31 0.6), medium level (0.61 0.90), medium-high level (0.91 1.2), and high level (>1.2). The spatiotemporal distribution map of 2005, 2009, 2013, and 2017 is shown in Fig. 5. As illustrated in Fig. 5, we can view significant spatial differences in agricultural eco-efficiency across counties.

In 2005 , the high agricultural eco-efficiency values were mainly distributed in counties characterized by a high agricultural economic level, such as Lishu, Gongzhuling, and Changling, with Lishu and Gongzhuling holding the highest ranks. Central counties with a medium-high efficiency level include Qian'an, Qianguo, Nongan, Dehui, Huadian, Yitong and Dongliao, while the counties with medium agricultural eco-efficiency are Fuyu, Yushu and Shuangliao. Other counties characterized by medium-low agricultural eco-efficiency, such as Taobei, Zhenlai, Taonan, Daan Tonghua and Yongji, are situated in the western and southeast region; such counties have great potential for improvement. In comparison, central and western counties rely on higher agricultural production input to support agricultural economic growth, which constrains the improvement in agricultural eco-efficiency to some content. 
In 2009, Yitong and Lishu County are the counties with a high level of agricultural ecoefficiency, while Qianan, Qianguo, Nong'an, Dehui, Yushu, Gongzhuling, Tonghua and Huadian have medium-high efficiency. The distribution of counties at the medium-high level is more concentrated in 2009 than in 2005. The counties with a medium-high level of agricultural ecoefficiency gradually expand from the central to the eastern agricultural production zone in Jilin, and their number increases. In addition, Shuangliao, Dongliao, and Panshi have a medium level of agricultural eco-efficiency, while Dongfeng and Liuhe have low agricultural eco-efficiency. The quantity and magnitude of counties with medium-low agricultural eco-efficiency change little.

In 2013, agricultural eco-efficiency decreased slightly, and its spatial agglomeration feature weakened, owing to regional differences in agricultural incentive policies. More than 15 counties had a medium-low level of agricultural efficiency. A possible reason for the decrease in agricultural eco-efficiency is that the execution of agricultural policy weakened. High-level efficiency can be found scattered across the province in Lishu, Yitong, and Huadian, while the counties with mediumhigh level efficiency are Qianguo, Nongan, Gongzhuling, Shuangliao, and Panshi. However, the distribution of counties at the medium-high level is more dispersed in 2013 than in 2009. In this year, there is only one county with medium-level efficiency, namely, Yushu County. The counties with medium-low agricultural eco-efficiency are Dongfeng and Tonghua.

In 2017, the county-level agricultural eco-efficiency presents a sharp increase with remarkable spatial variation. Yitong is the only county with a high level of eco-efficiency. The agricultural ecoefficiency in half of the counties is at the medium-high level, showing remarkable club convergence. In particular, the number of counties with agricultural eco-efficiency at the medium-high level gradually increases and the area of these counties expands from the areas east of Changchun to the central and western areas, while the counties with medium-low agricultural eco-efficiency gradually narrow in quantity and magnitude. The counties with medium-level eco-efficiency are scattered across the counties including Fuyu, Shulan, and Panshi And the number of counties with a medium level of agricultural eco-efficiency in 2017 increases compared with the number in 2013. The values for most counties in the central-southern JAPZ increase from the medium-low level and low level to the medium-high level in 2017. However, there are no longer any counties with low agricultural eco-efficiency. 
The four spatiotemporal distribution maps show that the spatial distribution has obvious coreperiphery characteristics; that is, the agricultural eco-efficiency of the central counties is generally higher than that of the southeast and northeast counties. In 2005, 2009, 2013, and 2017, there are 6 counties each year—including Nong'an, Huadian, Lishu, Yitong, Gongzhuling, and Qianguo — that achieve high agricultural eco-efficiency. The five counties with agricultural eco-efficiency values higher than 1 exhibit a growth pattern of low inputs, high outputs, and high agricultural ecoefficiency. The counties with a medium-low level of eco-efficiency are concentrated in the southeastern agricultural production zone in Jilin, while those with a low level continue to expand in quantity and are mainly concentrated in the central-eastern agricultural production zone.
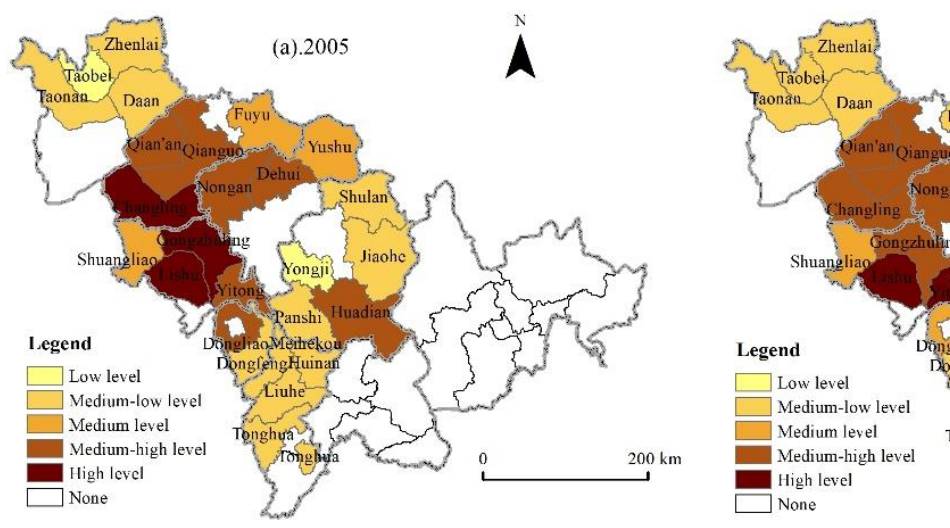

(b).2009
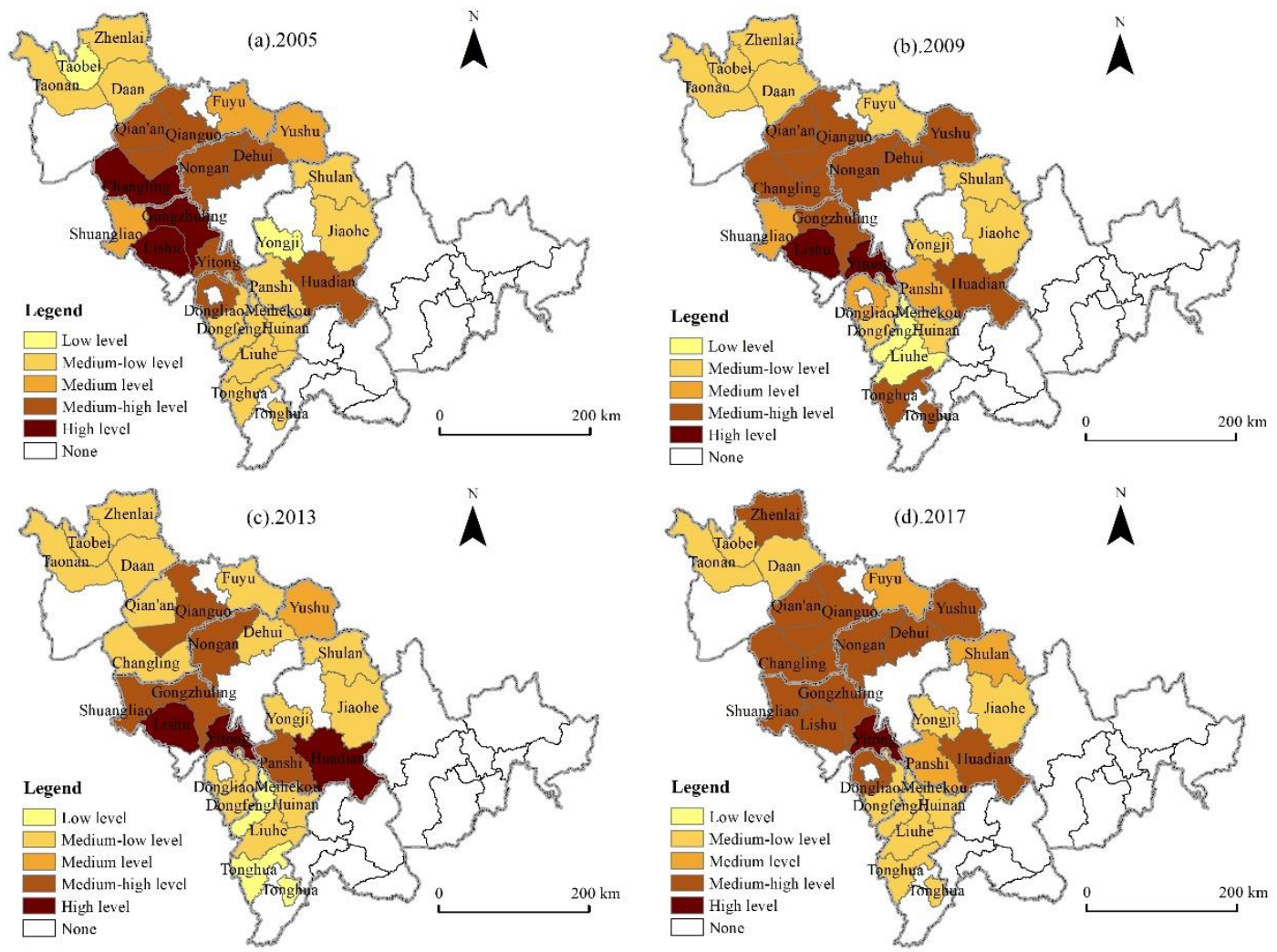

Fig. 5 The spatial distribution of agricultural eco-efficiency in the JAPZ in 2005, 2009, 2013, 2017

\subsection{Factors influencing agricultural eco-efficiency}

\subsubsection{Analysis of the panel data regression results}

A Pearson correlation test between the eight independent variables is conducted before the 
panel data regression is carried out. Table 4 illustrates the correlation matrices among variables. The test results show that the scores of the correlation matrices are small, which sufficiently confirms that all of the independent variables have weak correlations with each other. Therefore, the data for these eight independent variables are considered reliable for examining the influencing factors in the 26 counties studied in the panel data regression.

Table 4 Correlation matrices for independent variables.

\begin{tabular}{lllllllll}
\hline & $A M I$ & $M I$ & FFMS & ATEL & AEDL & AIS & UL & RRIL \\
\hline AMI & 1 & & & & & & & \\
MI & 0.267 & 1 & & & & & & \\
FFMS & 0.135 & -0.001 & 1 & & & & & \\
ATEL & 0.175 & -0.002 & -0.108 & 1 & & & & \\
AEDL & -0.016 & -0.048 & 0.340 & 0.136 & 1 & & & \\
AIS & 0.348 & 0.056 & 0.360 & -0.148 & -0.016 & 1 & & \\
UL & 0.222 & 0.101 & 0.233 & 0.071 & 0.556 & 0.206 & 1 & \\
RRIL & 0.382 & 0.085 & 0.044 & 0.340 & 0.380 & -0.138 & 0.113 & 1 \\
\hline
\end{tabular}

Static panel data models include several main types, namely, the mixed model (MM), fixed effect model (FEM), and random effect model (REM). To determine which model is most appropriate, the $\mathrm{F}$ test and Hausman test are required before construction of the panel data regression model. The F test is applied to determine whether to adopt the MM or the FEM. The Hausman test is applied to determine whether to select the FEM or the REM. The panel data regression model is conducted using the EViews 10.0 software tool. The F test statistic value is 20.293 , and its p value approaches 0.00 , which indicates that the null hypothesis of the MM can be rejected, leading us to accept the FEM. Additionally, the test statistic of the Hausman test is 70.683 , and its p value approaches 0.00 , which indicates that the null hypothesis of the REM is rejected and likewise suggests the FEM as appropriate. Therefore, the FEM should be established as the appropriate model according to the results of $\mathrm{F}$ test and Hausman test. The $\mathrm{R}^{2}$ of the FEM and REM are 0.688 and 0.454 , respectively, which illustrates that the fit degree of the panel data regression model with fixed effects is more better than that the model with random effects. The FEM regression results of the eight independent variables on agricultural eco-efficiency are shown in Table 5 and are further 
applied to analyze the influencing factors of agricultural eco-efficiency.

The results of the panel data regression set out in Table 5 indicate that all variables, with the exception of the multiple-crop index (MI), agricultural industrial structure (AIS), and rural resident income level (RRI), pass the $10 \%$ significance level. Specifically, the agricultural technology extension level, agricultural economic development level, and agricultural industry structure all have positive correlations with agricultural eco-efficiency in the 26 counties during the observation period at the $1 \%, 1 \%$ and $5 \%$ levels, respectively. Conversely, both agricultural mechanization intensity and urbanization level have negative correlations with agricultural eco-efficiency at the 5\% and $1 \%$ levels.

\subsubsection{Estimation results analysis}

The estimated coefficient of agricultural machinery intensity (AMI) is negative and significant at the $1 \%$ level, implying that agricultural machinery intensity suppresses the improvement in agricultural eco-efficiency as a whole. This finding is associated with the large amount of fossil fuel such machinery consumes, which further increases the negative environmental impact in the agricultural production process. Specifically, agricultural machinery intensity affects the agricultural resource inputs and increases the agricultural production efficiency but also leads to a large increase in the consumption of energy resources, such as diesel oil, with the consequence that the agricultural machinery intensity does not promote the improvement in agricultural ecoefficiency. That is, higher agricultural machinery intensity means more diesel oil consumption and more carbon emissions, which is not beneficial for agricultural eco-efficiency. Thus, the development of agricultural mechanization must be controlled accordingly to the intensity and scale of mechanization.

The correlation coefficient between family farmland management scale (FFMC) and agricultural eco-efficiency is positive and significant at the $1 \%$ level, indicating that the increase in family farmland management scale is conducive to the increase in agricultural eco-efficiency. This study offers several explanations for this finding. On the one hand, rich arable land resources and complete arable land plots in the JAPZ provide superior conditions for the development of largescale agricultural operations. The development of new management entities engaged in large-scale 
farmland operations is relatively mature. On the other hand, with a large amount of arable land gradually concentrated on cultivation experts, moderate scale benefits of agriculture gradually appeared and farmers with large farmland management scale can use more advanced and efficient agricultural technology than farmers with small-scale farmland for agricultural production, which can improve the agricultural eco-efficiency. Therefore, when the family farmland management scale is large, the agricultural eco-efficiency is high.

Additionally, the correlation coefficient between agricultural technology extension level (ATEL) and agricultural eco-efficiency is positive and significant at the $1 \%$ level, indicating that improvement in the agricultural technology extension level tends to intensify the increase in agricultural eco-efficiency. This is because agricultural professional and technical personnel can guide farmers to implement environmentally friendly agricultural production methods, which is conducive to driving productivity and optimizing the production process via the technology effect. In addition, as displayed in Table 5, the estimated correlation coefficient is 0.093 , passing the significance test, which demonstrates that this indicator can sufficiently influence agricultural ecoefficiency, but the effect is not obvious. This result directly confirms that environmental technology and environmental management skills can bring about the improvement in overall agricultural ecoefficiency. That is, counties with more agricultural technology support for agriculture have the ability to curb negative impacts on the agricultural ecological environment.

The correlation coefficient of agricultural economic development level (AEDL) and agricultural eco-efficiency positively pass the significance test at the $1 \%$ level, indicating that an increase in agricultural economic development level can accelerate the increase in agricultural ecoefficiency. Numerically, the correlation coefficient of AEDL, reaching 0.633, is larger than the correlation coefficients of the other variables, which illustrates that the agricultural economic development level occupies the leading position in the development of agricultural eco-efficiency, and the "economic attributes" are most important for agricultural eco-efficiency. A possible reason for the positive effect of agricultural economic development level on agricultural eco-efficiency is that there are large number of national agricultural counties in the JAPZ. The obvious agricultural development scale effect can not only stimulate the expansion of agricultural production but also improve farming methods and optimize agricultural materials for the high-quality development of 
agricultural production. In addition, the counties with a high agricultural economic development level are likely to achieve a balance between agricultural production and the ecological environment with the growth of agricultural intensification and specialization, which is conducive to increasing agricultural eco-efficiency.

The correlation coefficient of urbanization level (UL) indicates that this indicator has a significant negative influence on agricultural eco-efficiency at the $1 \%$ level, which indicates that the increase in urbanization level can hinder agricultural eco-efficiency. Moreover, the negative correlation coefficient of urbanization level is larger than that of agricultural machinery intensity in terms of the impact degree. This finding illustrates that among the selected variables, the urbanization level is the greatest hindrance to agricultural eco-efficiency. The influence of the urbanization level on agricultural eco-efficiency is similar to that of agricultural machinery intensity. Since the eleventh five-year plan period, surplus labor has flowed between urban and rural areas, accompanying rapid urban construction. As a consequence of the surplus labor transfer to urban areas, urban development has also squeezed out the input of labor, capital and other factors required for agricultural production, leading to changes in the employment structure. The laborers who remain in the countryside have to use more agricultural machinery to compensate for the loss in labor via the substitution effect, which can cause the deterioration of the agricultural ecological environment. It is not difficult to understand that when the urbanization level increases, the agricultural eco-efficiency may finally decrease.

Table 5 The results of the panel data regression.

\begin{tabular}{lllllll}
\hline \multirow{2}{*}{ Variables } & \multicolumn{2}{c}{ MM } & \multicolumn{2}{c}{ FEM } & \multicolumn{2}{c}{ REM } \\
\cline { 2 - 6 } & Coefficient & t-statistic & Coefficient & t-statistic & Coefficient & t-statistic \\
\hline AMI & $-0.307^{* * *}$ & -3.972 & $-0.228^{* * *}$ & -2.878 & $-0.312^{* * *}$ & -4.205 \\
MI & -0.09 & -0.365 & 0.183 & 0.929 & $-0.088^{*}$ & -0.388 \\
ATEL & $0.127^{*}$ & 1.714 & $0.128^{*}$ & 1.661 & $0.123^{* * *}$ & 1.727 \\
AEDL & $0.087^{* * *}$ & 3.431 & $0.093^{* * *}$ & 4.510 & $0.087^{* * *}$ & 3.722 \\
AIS & $0.389^{* * *}$ & 5.451 & $0.633^{* * *}$ & 8.728 & $0.392^{* * *}$ & 5.850 \\
UL & $-0.443^{* * *}$ & -6.265 & -0.134 & -1.389 & $-0.428^{* * *}$ & -4.268 \\
$R R I L$ & $-0.525^{* * *}$ & -8.722 & $-0.483^{* * *}$ & -9.200 & $-0.524^{* * *}$ & -9.431 \\
& -0.069 & -0.975 & 0.104 & 0.713 & -0.065 & -0.935
\end{tabular}




\begin{tabular}{|c|c|c|c|c|c|}
\hline$c$ & 0.176 & -0.162 & -0.234 & -4.033 & 0.126 \\
\hline $\mathrm{R}^{2}$ & 0.454 & & 0.688 & & 0.454 \\
\hline Adjusted $\mathrm{R}^{2}$ & 0.442 & & 0.654 & & 0.440 \\
\hline
\end{tabular}

\section{Conclusion and policy suggestions}

\subsection{Conclusion}

During the observed period of 2005-2017, the average value of agricultural eco-efficiency in the JAPZ exhibited a rising trend with fluctuations. The agricultural eco-efficiency of each county could still improve in terms of resource conservation and environmental protection, although it is still at a medium level overall. This study also reveals that there are significant spatial differences in agricultural eco-efficiency in the JAPZ. The agricultural eco-efficiency in the region presents a spatial pattern that progresses from the core to the periphery. Specifically, central counties usually have higher agricultural eco-efficiency than southeastern and northwestern counties. Nong'an, Huadian, Lishu, Yitong, Gongzhuling, and Qianguo achieved high agricultural eco-efficiency in 2005, 2009, 2013, and 2017. Since the change in agricultural eco-efficiency results from the complex interaction of various factors, this study further considers various influencing factors that lead to the change in agricultural eco-efficiency. The panel data regression estimation results indicate that the agricultural technology extension level, agricultural economic development level, agricultural industry structure, agricultural mechanization intensity and urbanization level have close correlations with agricultural eco-efficiency. The agricultural economic development level occupies the leading position in the development of agricultural eco-efficiency while urbanization level has greatest hindrance to agricultural eco-efficiency.

\subsection{Policy suggestions}

According to the aforementioned contributing factors, differentiated suggestions are proposed for policy makers. First, the results show that agricultural mechanization intensity constrains agricultural eco-efficiency. Governments in the JAPZ should control the intensity and scale of mechanization, eliminate agricultural machinery with high energy consumption and low production 
efficiency and adopt new environmentally friendly technology to maintain the stability of the agricultural ecological environment. Second, the correlation coefficient of technological progress is not high, but it is large enough to have an impact on agricultural eco-efficiency, which indicates that its key role in the improvement in agricultural eco-efficiency should not be neglected, although the agricultural technology extension level exerts a slight effect on agricultural eco-efficiency. Technical training and professional skills should be provided for farmers in the JAPZ to help them better master relevant green, sustainable agricultural technologies. Third, the results show that the family farmland management scale can accelerate the improvement in agricultural eco-efficiency, which is conducive to the adoption of new agricultural technologies. A moderate scale of agricultural eco-efficiency can relieve the pressure of high energy consumption on cultivated land. Fourth, among the selected variables, the agricultural economic development level is the strongest positive factor driving agricultural eco-efficiency, implying that the scale expansion and total growth of the agricultural economy are still the key ways to promote agricultural eco-efficiency. Hence, the continuous improvement of the agricultural economic development level is still one of the vital ways to increase agricultural eco-efficiency. Taking ecological priorities and green development as the guidance, the extensive agricultural production and management model should be transformed, the development methods should be optimized, circular and ecological agriculture should be developed, and the sustainable use of agricultural resources should be promoted to support high-quality agricultural development in the JAPZ. Finally, the urbanization level exerts a negative impact on agricultural eco-efficiency, indicating that ecology-oriented agricultural subsidies should be improved and a high-efficiency compensation mechanism be established to stimulate the enthusiasm for agricultural production in the JAPZ.

There is an urgent practical need for research on agricultural eco-efficiency under resource and environmental constraints. This study intends to narrow the gap in the literature on agricultural ecoefficiency, and several limitations remain that deserve in-depth attention in future research. In fact, agricultural eco-efficiency is also affected by natural factors, such as climate, soil properties and the natural environment, which influence the input and output of agricultural production to some extent. Due to the limited availability of data regarding natural factors, this study investigated only socioeconomic factors. When more natural data are publicly available, the spatial dimension and 
the indicators applied in our study could be improved upon to enable more comprehensive modeling. Moreover, the influencing factors of agricultural eco-efficiency could be further investigated from the perspective of national agricultural production zones to help accomplish China's future carbon emission reduction targets ahead of schedule and achieve the green agricultural transition.

Acknowledgments The authors gratefully acknowledge all the reviewers and editors for their insightful comments.

Data Availability Statement Some or all data, models, or code that support the findings of this study are available from the corresponding author upon reasonable request.

Author's Contributions Methodology: Guo Yanhua; Software: Guo Yanhua; Writing- Original draft: Guo Yanhua; Conceptualization:Tong Lianjun, Mei Lin; Funding acquisition: Tong Lianjun; Writing - review \& editing: Mei Lin.

Funding The research received financial support from the National Natural Science Foundation of China (No. 41771138).

\section{Compliance with ethical standards}

Conflict of interest The authors declare no conflicts of interest.

\section{Ethical approval Not applicable.}

Consent to participate Not applicable.

Consent to publication Not applicable.

\section{References}

Ali S, Gucheng L, Ying L, Ishaq M, Shah T (2019) The relationship between carbon dioxide emissions, economic growth and agricultural production in Pakistan: An autoregressive distributed lag analysis. Energies 12(24), 4644

Banker R D, Charnes A, Cooper W W (1984) Some models for estimating technical and scale inefficiencies in data envelopment analysis. Manage. Sci. 30(9), 1078-1092

Baum R, Bieńkowski J (2020) Eco-Efficiency in measuring the sustainable production of agricultural crops. Sustainability 12(4), 1418 
611 Burnett R D, Hansen D R (2008) Ecoefficiency: Defining a role for environmental cost management.

612 Account. Organ. Soc. 33(6), 551-581

613 Camarero M, Castillo J, Picazo-Tadeo A J, Tamarit C (2013) Eco-efficiency and convergence in

614 OECD countries. Environ. Resour. Econ. 55(1), 87-106

615 Charnes A, Cooper W W, Rhodes E (1978) Measuring the efficiency of decision making units. Eur.

$616 \quad$ J. Oper. Res. 2(6), 429-444

617 Chen N, Xu L, Chen Z, Lund H, Kaiser M J (2017) Environmental efficiency analysis of the Yangtze

618 River Economic Zone using super efficiency data envelopment analysis (SEDEA) and Tobit models.

619 Energy 134, 659-671

620 Coluccia B, Valente D, Fusco G, De Leo F, Porrini D (2020) Assessing agricultural eco-efficiency

621 in Italian Regions. Ecol. Ind. 116, 106483

622 Czyżewski B, Matuszczak A, Muntean A (2019) Approaching environmental sustainability of 623 agriculture: environmental burden, eco-efficiency or eco-effectiveness. Agr. Econ. 65(7), 299-306

624 Czyżewski B, Matuszczak, Grzelak A, Guth M, Majchrzak A (2020) Environmental sustainable 625 value in agriculture revisited: How does Common Agricultural Policy contribute to eco-efficiency? 626 Sustain. Sci. 1-16

627 Deng X, Gibson J (2019) Improving eco-efficiency for the sustainable agricultural production: A case study in Shandong, China. Technol. Forecast. Soc. Chang. 144, 394-400

629 Dubey A, Lal R (2009) Carbon footprint and sustainability of agricultural production systems in 630 Punjab, India, and Ohio, USA. J. Crop Improv. 23(4), 332-350

631 Fan J, Li P (2009) The scientific foundation of major function oriented zoning in China. J. Geog. 632 Sci. 19(5), 515

633 Fan J, Sun W, Zhou K, Chen D (2012) Major function oriented zone: New method of spatial 634 regulation for reshaping regional development pattern in China. Chin. Geog. Sci. 22(2), 196-209

635 Färe R, Grosskopf S, Lovell C K, Pasurka C (1989) Multilateral productivity comparisons when 636 some outputs are undesirable: a nonparametric approach. Rev. Econ. Stat. 71, 90-98

637 Gkiza I G, Nastis S A (2017) Health and women's role in agricultural production efficiency. Appl. 638 Econ. Perspect. Policy 39(3), 428-440

639 Gössling S, Peeters P, Ceron J P, Dubois G, Patterson T, Richardson R B (2005) The eco-efficiency 
of tourism. Ecol. Econ. 54(4), 417-434

Gómez-Limón J A, Picazo-Tadeo A J, Reig-Martínez E (2012) Eco-efficiency assessment of olive farms in Andalusia. Land Use Policy 29(2), 395-406

Grossman G M, Krueger A B (1995) Economic growth and the environment. Quart. J. Econ. 110(2): $353-377$

Han H, Zhang X (2020) Exploring environmental efficiency and total factor productivity of cultivated land use in China. Sci. Total Environ. 138434

He J, Wan Y, Feng L, Ai J, Wang Y,] (2016) An integrated data envelopment analysis and emergybased ecological footprint methodology in evaluating sustainable development, a case study of Jiangsu Province, China. Ecol. Ind. 70, 23-34

Huang J, Xia J, Yu Y, Zhang N (2018) Composite eco-efficiency indicators for China based on data envelopment analysis. Ecol. Ind. 85, 674-697

Huang X, Xu X, Wang Q, Zhang L, Gao X, Chen L (2019) Assessment of agricultural carbon emissions and their spatiotemporal changes in China, 1997-2016. Int. J. Environ. Res. Public Health 16(17), 3105

Jin G, Li Z, Deng X, Yang J, Chen D, Li W (2019) An analysis of spatiotemporal patterns in Chinese agricultural productivity between 2004 and 2014. Ecol. Ind. 105, 591-600

Li H, Fang K, Yang W, Wang D, Hong X (2013) Regional environmental efficiency evaluation in China: Analysis based on the Super-SBM model with undesirable outputs. Math. Comput. Modell. 58(5-6), 1018-1031

Lio M C, Hu J L (2009) Governance and agricultural production efficiency: a cross-country aggregate frontier analysis. J. Agr. Econ. 60(1), 40-61

Liu Q, Wang S, Li B, Zhang W (2020a) Dynamics, differences, influencing factors of eco-efficiency in China: A spatiotemporal perspective analysis. J. Environ. Manage. 264, 110442

Liu J, Zhang J, Fu Z (2017) Tourism eco-efficiency of Chinese coastal cities-Analysis based on the DEA-Tobit model. Ocean. Coastal. Manage. 148, 164-170

Liu Y, Zou L, Wang Y (2020b) Spatial-temporal characteristics and influencing factors of agricultural eco-efficiency in China in recent 40 years. Land Use Policy 97, 104794

Ma X, Wang C, Yu Y, Li Y, Dong B, Zhang X, ..., Gu Y (2018a) Ecological efficiency in China 
and its influencing factors - a super-efficient SBM metafrontier-Malmquist-Tobit model study. Environ. Sci. Pollut. Res. 25(21), 20880-20898.

Ma X, Li Y, Zhang X, Wang C, Li Y, Dong B, Gu Y (2018b) Research on the ecological efficiency of the Yangtze River Delta region in China from the perspective of sustainable development of the economy-energy-environment (3E) system. Environ. Sci. Pollut. Res. 25(29), 29192-29207.

Moutinho V, Madaleno M, Macedo P (2020) The effect of urban air pollutants in Germany: ecoefficiency analysis through fractional regression models applied after DEA and SFA efficiency predictions. Sust. Cities Soc. 102204

Peng H, Zhang J, Lu L, Tang G, Yan B, Xiao X, Han Y (2017) Eco-efficiency and its determinants at a tourism destination: A case study of Huangshan National Park, China. Tourism Manage. 60, 201-211

Picazo-Tadeo A J, Gómez-Limón J A, Reig-Martínez E (2011) Assessing farming eco-efficiency: a data envelopment analysis approach. J. Environ. Manage. 92(4), 1154-1164

Reith C C, Guidry M J (2003) Eco-efficiency analysis of an agricultural research complex. J. Environ. Manage. 68(3), 219-229

Ren Y, Fang C, Lin X, Sun S, Li G, Fan B (2019) Evaluation of the eco-efficiency of four major urban agglomerations in coastal eastern China. J. Geog. Sci. 29(8), 1315-1330

Saravia-Matus S L, Hörmann P A, Berdegué J A (2019) Environmental efficiency in the agricultural sector of Latin America and the Caribbean 1990-2015: Are greenhouse gas emissions reducing while agricultural production is increasing?. Ecol. Ind. 102, 338-348

Schaltegger S, Sturm A (1990) Ökologische rationalität: Ansatzpunkte zur ausgestaltung von ökologieorientierten management instru-menten. Die Unternehmung, 44(4): 273-290.

Sinkin C, Wright C J, Burnett R D (2008) Eco-efficiency and firm value. J. Account. Public Policy. 27(2), 167-176

Tian Y, Zhang J B, He Y Y (2014) Research on spatial-temporal characteristics and driving factor of agricultural carbon emissions in China. J. Integr. Agric. 13(6), 1393

Todorovic M, Mehmeti A, Scardigno A (2016) Eco-efficiency of agricultural water systems: Methodological approach and assessment at meso-level scale. J. Environ. Manage. 165, 62-71

Tone K (2001) A slacks-based measure of efficiency in data envelopment analysis. Eur. J. Oper. 
Res. 130(3), 498-509

Tone K (2002) Slacks-based measure of super-efficiency in data envelopment analysis. Eur. J. Oper. Res. 143(1), 32-41

Vlontzos G, Niavis S, Manos B (2014) A DEA approach for estimating the agricultural energy and environmental efficiency of EU countries. Renew. Sust. Energ. Rev. 40, 91-96 Wang Y, Fan J (2020) Multi-scale analysis of the spatial structure of China's major function zoning. J. Geog. Sci. 30(2), 197-211

West T O, Marland G (2002) A synthesis of carbon sequestration, carbon emissions, and net carbon flux in agriculture: comparing tillage practices in the United States. Agric. Ecosyst. Environ. 91(13), $217-232$

Xing Z, Wang J, Zhang J (2018) Expansion of environmental impact assessment for eco-efficiency evaluation of China's economic sectors: An economic input-output based frontier approach. Sci. Total Environ. 635, 284-293

Yang L, Yang Y (2019) Evaluation of eco-efficiency in China from 1978 to 2016: Based on a modified ecological footprint model. Sci. Total Environ. 662, 581-590

Yin K, Wang R, An Q, Yao L, Liang J (2014) Using eco-efficiency as an indicator for sustainable urban development: A case study of Chinese provincial capital cities. Ecol. Ind. 36, 665-671

Zhang B, Bi J, Fan Z, Yuan Z, Ge J (2008) Eco-efficiency analysis of industrial system in China: A data envelopment analysis approach. Ecol. Econ. 68(1-2), 306-316

Zhou C, Shi C, Wang S, Zhang G (2018) Estimation of eco-efficiency and its influencing factors in Guangdong province based on Super-SBM and panel regression models. Ecol. Ind. 86, 67-80 Zhou Y, Kong Y, Zhang T (2020) The spatial and temporal evolution of provincial eco-efficiency in China based on SBM modified three-stage data envelopment analysis. Environ. Sci. Pollut. Res. 27(8), 8557-8569 


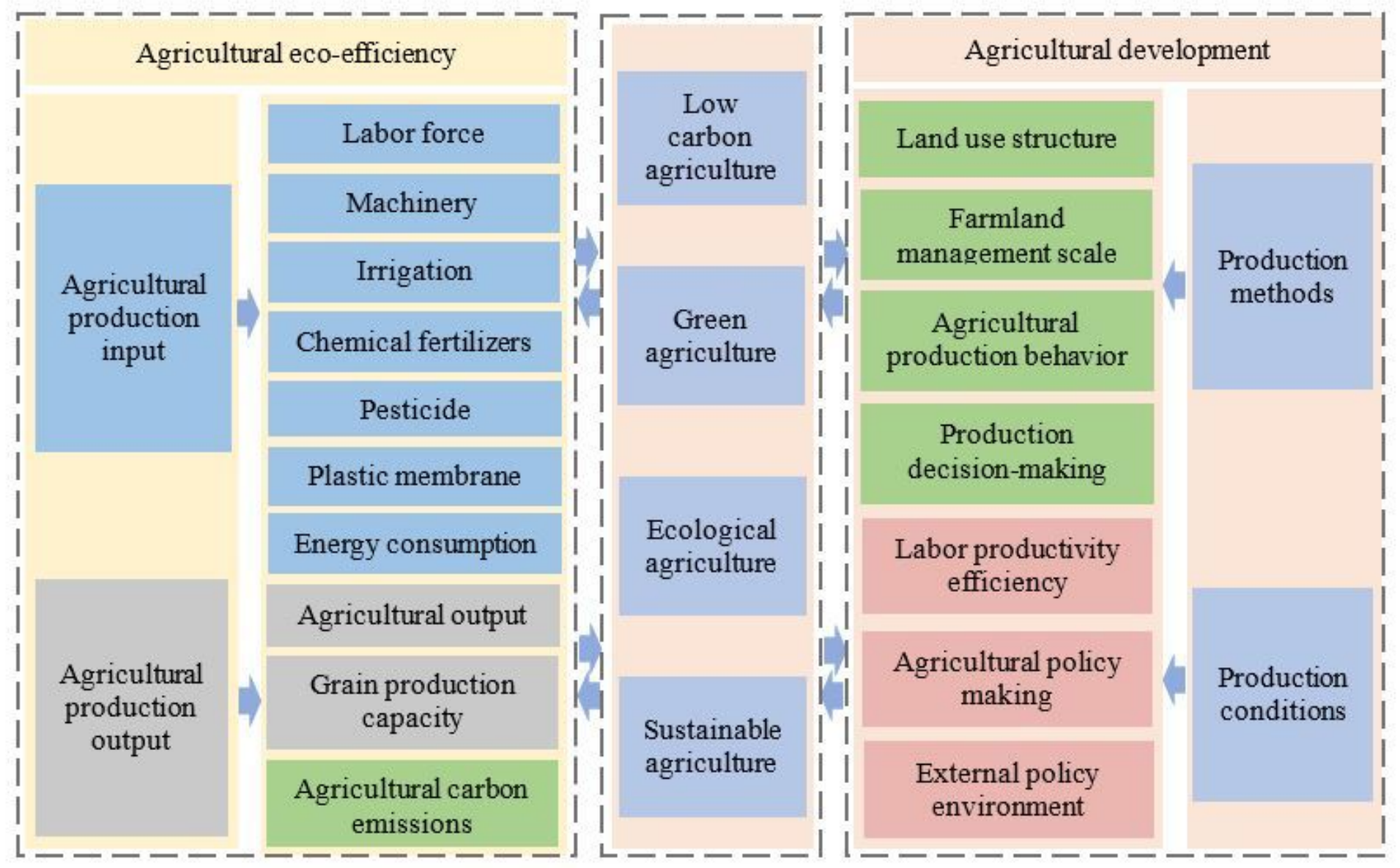

\section{Figure 1}

An analytical framework illustrating the interactions between agricultural production and agricultural ecoefficiency.
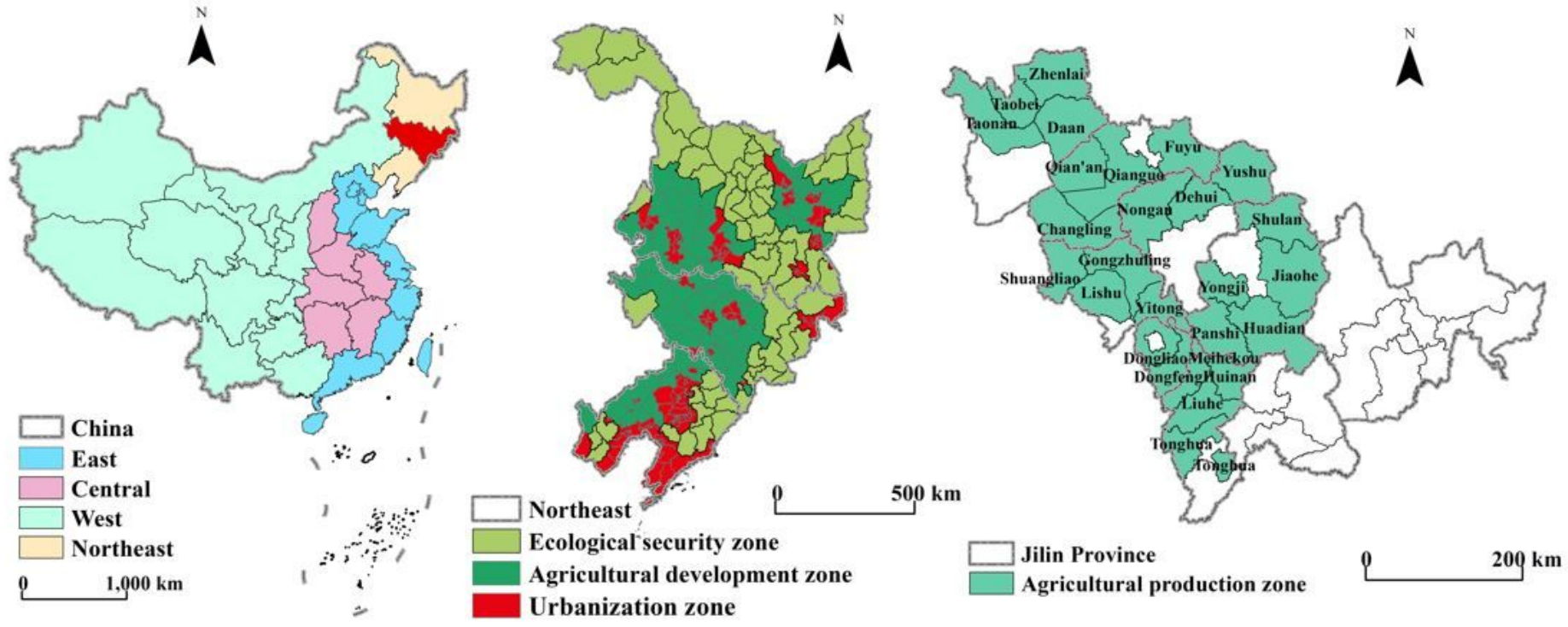
Figure 2

Map of the empirical study area. Note: The designations employed and the presentation of the material on this map do not imply the expression of any opinion whatsoever on the part of Research Square concerning the legal status of any country, territory, city or area or of its authorities, or concerning the delimitation of its frontiers or boundaries. This map has been provided by the authors.

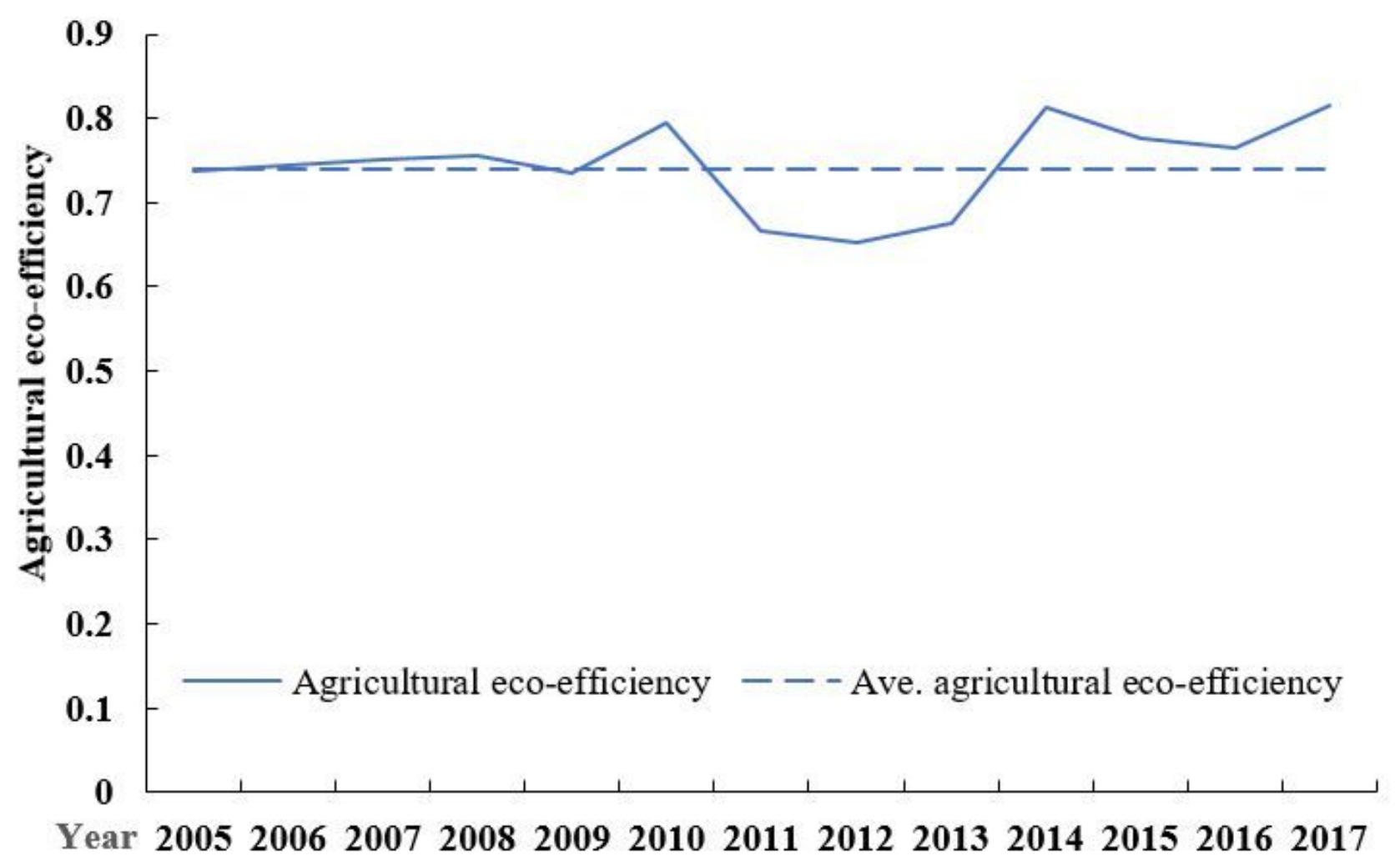

\section{Figure 3}

The agricultural eco-efficiency change trend of the JAPZ, 2005-2017. 


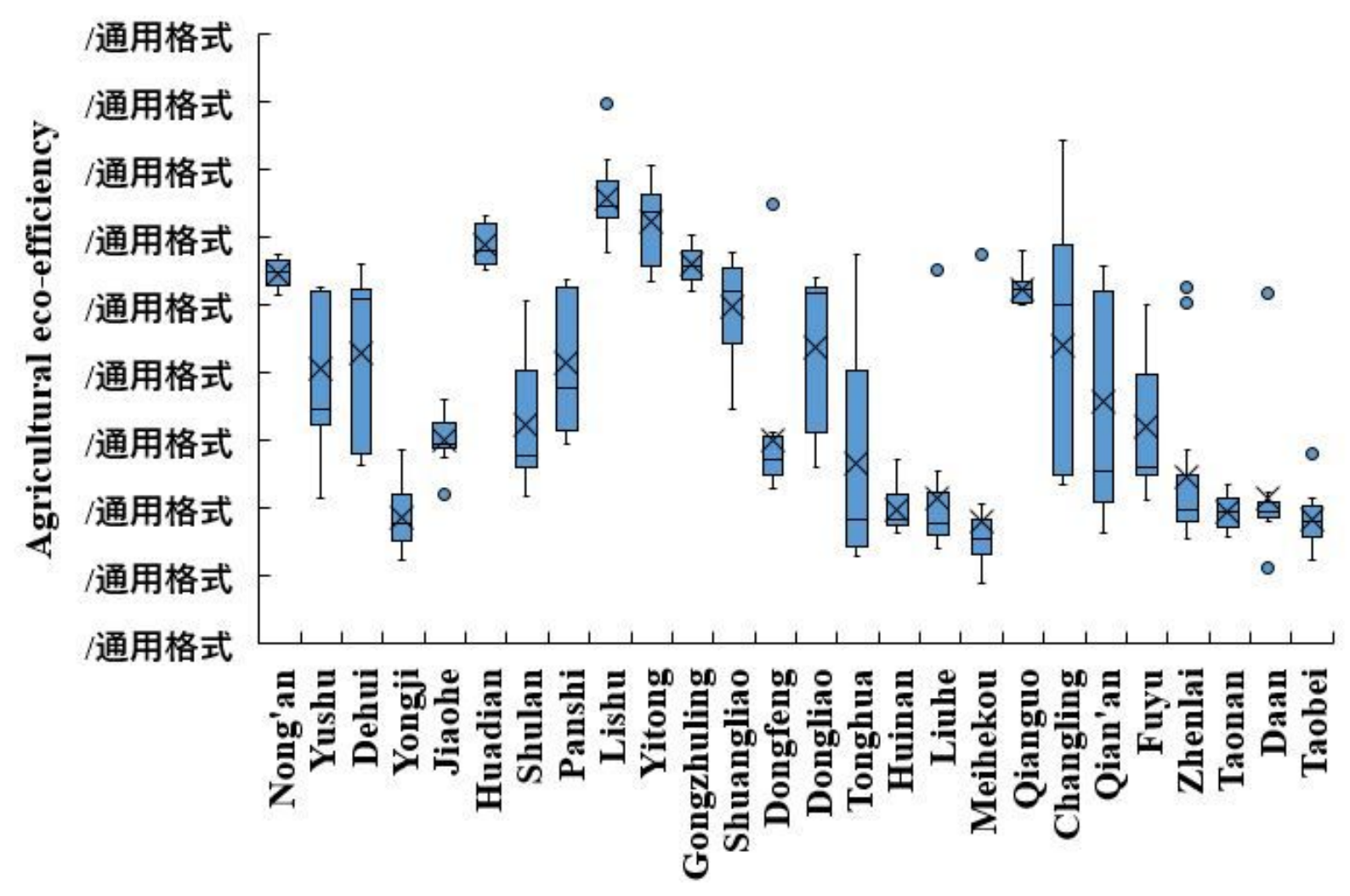

Figure 4

Boxplot of the agricultural eco-efficiency of 26 counties in the JAPZ, 2005-2014. 

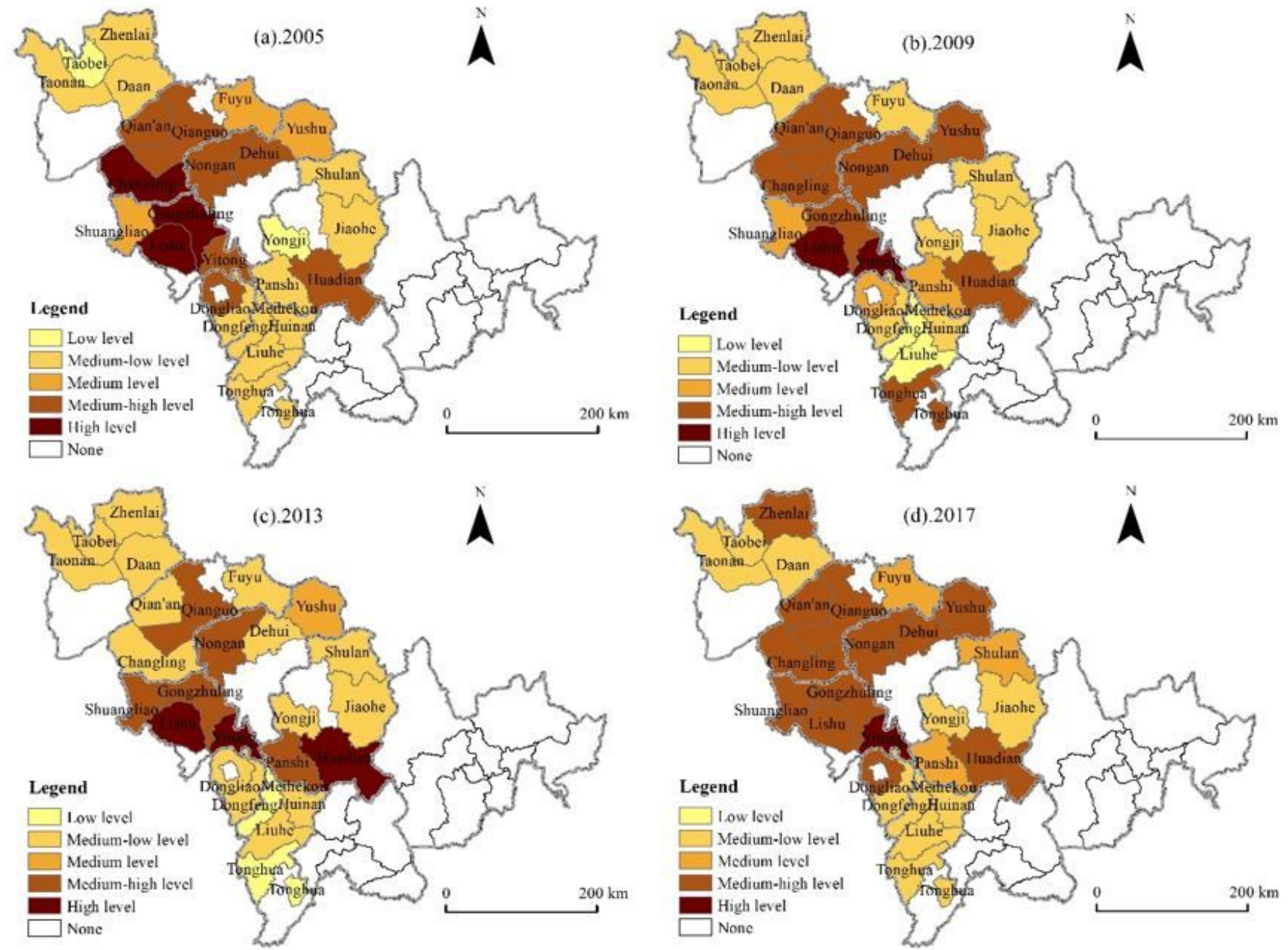

(d). 2017
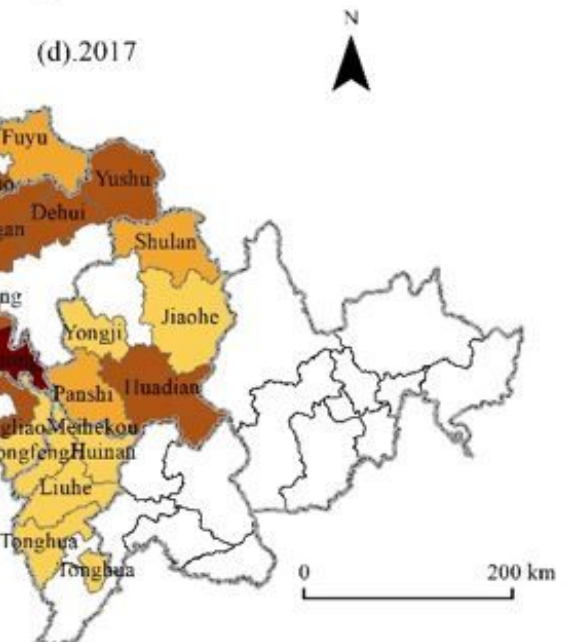

\section{Figure 5}

The spatial distribution of agricultural eco-efficiency in the JAPZ in 2005, 2009, 2013, 2017 Note: The designations employed and the presentation of the material on this map do not imply the expression of any opinion whatsoever on the part of Research Square concerning the legal status of any country, territory, city or area or of its authorities, or concerning the delimitation of its frontiers or boundaries. This map has been provided by the authors. 